\title{
First Results of a GNSS-R Experiment From a Stratospheric Balloon Over Boreal Forests
}

\author{
Hugo Carreno-Luengo, Member, IEEE, Adriano Camps, Fellow, IEEE, Jorge Querol, Student Member, IEEE, \\ and Giuseppe Forte, Member, IEEE
}

\begin{abstract}
The empirical results of a global navigation satellite systems reflectometry (GNSS-R) experiment onboard the Balloon EXperiments for University Students (BEXUS) 17 stratospheric balloon performed north of Sweden over boreal forests show that the power of the reflected signals is nearly independent of the platform height for a high coherent integration time $T_{c}=20 \mathrm{~ms}$. This experimental evidence shows a strong coherent component in the forward scattered signal, as compared with the incoherent component, that allows to be tracked. The bistatic coherent reflectivity is also evaluated as a function of the elevation angle, showing a decrease of $\sim 6 \mathrm{~dB}$ when the elevation angle increases from $35^{\circ}$ to $70^{\circ}$. The received power presents a clearly multimodal behavior, which also suggests that the coherent scattering component may be taking place in different forest elements, i.e., soil, canopy, and through multiple reflections canopy-soil and soil-trunk. This experiment has provided the first GNSS-R data set over boreal forests. The evaluation of these results can be useful for the feasibility study of this technique to perform biomass monitoring that is a key factor to analyze the carbon cycle.
\end{abstract}

Index Terms-Boreal forests, global navigation satellite systems reflectometry (GNSS-R), stratospheric balloon.

\section{INTRODUCTION}

$\mathbf{I}$ N THE early 1990s, the concept of global navigation satellite systems reflectometry (GNSS-R) was first proposed by Martín-Neira for mesoscale ocean altimetry [1] after the evidence that GPS navigation signals could be collected after being scattered on the sea surface [2]. Newer applications of GNSS-R include wind speed measurements [3], [4], ice altimetry [5], soil moisture, and vegetation determination [6]. Several experiments have been carried out to analyze the performance of different GNSS-R techniques, i.e., conventional GNSS-R (cGNSS-R) (GPS L1 C/A) (e.g., see [7]), interferometric GNSS-R (iGNSS-R) (GPS L1 C/A, P(Y), and M) [8], and reconstructed-code GNSS-R (rGNSS-R) [GPS L1 and L2 P(Y)] [9].

Manuscript received November 10, 2014; revised March 4, 2015, June 25, 2015, and November 9, 2015; accepted November 18, 2015. Date of publication December 18, 2015; date of current version March 25, 2016. This work was supported in part by the European Union's Seventh Framework Programme for research, technological development, and demonstration through agreement "European GNSS-R Environmental Monitoring" under Grant FP7-607126-EGEM (available online: http://www.e-gem.eu) and in part by the Spanish Ministerio de Ciencia e Innovación (MICINN) through the project "Aplicaciones avanzadas en radio ocultaciones y dispersometría utilizando señales GNSS y otras señales de oportunidad" under Grant AYA2011-29183-C02-01.

The authors are with the Remote Sensing Laboratory, Department of Signal Theory and Communications, Universitat Politécnica de Catalunya, 08034 Barcelona, Spain (e-mail: carrenyo@ieec.cat; camps@tsc.upc.edu; jorge. querol@tsc.upc.edu; giuseppe.forte@tsc.upc.edu).

Color versions of one or more of the figures in this paper are available online at http://ieeexplore.ieee.org.

Digital Object Identifier 10.1109/TGRS.2015.2504242
The potential of GNSS-R to provide soil moisture measurements over land was first assessed in 2000 [10]. As compared with GPS reflections over the ocean, the main differences identified are in the spatial and temporal variability of the soil dielectric constant, the surface roughness, and the vegetation cover. Over land, the width of the correlated waveform (WF $)^{1}$ is much narrower than over the ocean surface, and the variability of the moisture is clearly manifested in the peak power of the WF [10]. In 2008, the multipath signal collected by geodetic GPS receivers was used to infer soil moisture information from the fluctuations of the signal-to-noise ratio (SNR) [11]. In 2009, the interference pattern technique (IPT) was proposed [12] for soil moisture measurements using linear polarization antennas pointing toward the horizontal direction to increase the amplitude of the signal that is being degraded by fading noise. Then, the IPT was proposed for vegetation height retrieval [13]. In 2012, a similar technique as in [11] was proposed [14] to infer vegetation growth. The retrieval method relied on amplitude changes. In 2009, field experimental results showed the capabilities of GNSS-R polarimetric observations as a remote sensing tool for agricultural applications from ground-based receivers [15], [16], and a simulator was developed to interpret these results [17]. The framework to analyze the coherent scattering over soil was established by Fung and Eom [18], and the scattering as a function on the antenna beamwidth, the incident and scattering angles, and the distance from the antenna to the target was evaluated in [19].

In July 2014, the TechDemoSat-1 mission of Surrey Satellite Technology Ltd., was successfully launched [20]. In addition, at least four other spaceborne missions are currently approved or "under study," i.e., NASA's Cyclone GNSS (CyGNSS) [21], ESA's GNSS-R, Radio Occultation, and Scatterometry Experiment Onboard the International Space Station (GEROS-ISS) [22], ESA's Passive Reflectometry and Interferometry System In-Orbit Demonstrator (PARIS-IoD) [23], and ${ }^{3}$ Cat-2 [24].

This paper presents the results of an experiment carried out in the Swedish National Space Board (SNSB)/ESA-sponsored Balloon EXperiments for University Students (BEXUS) 17 stratospheric balloon over boreal forests north of Sweden on October 10, 2013, as a proof of concept of the payload of the ${ }^{3}$ Cat-2 mission [24]. Section II describes the experimental setup. Section III presents the study of the scattered electromagnetic fields over the boreal forests using experimental data.

\footnotetext{
${ }^{1}$ The WF is the temporal correlation of the received reflected signal with either a locally generated replica of the transmitted signal (cGNSS-R) or the direct signal itself (iGNSS-R).
} 


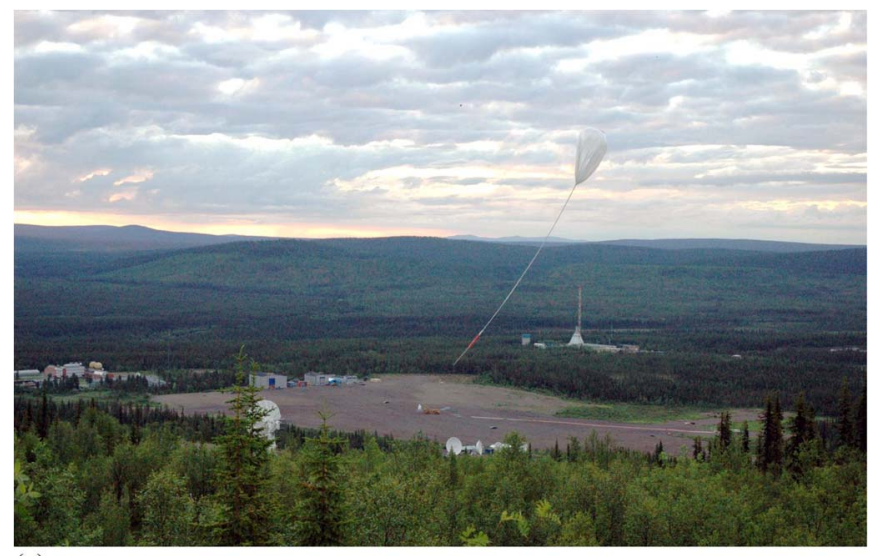

(a)

(b)

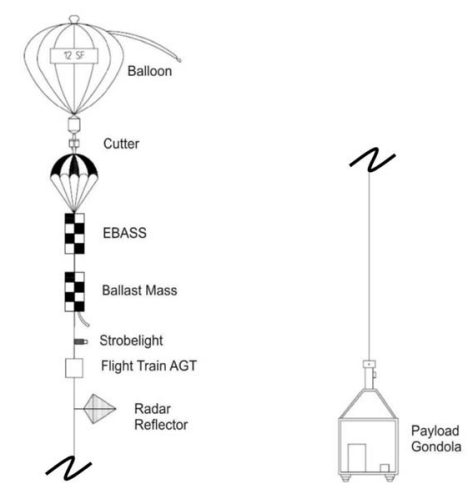

Fig. 1. (a) Stratospheric balloon during takeoff at the Esrange Space Center. (b) Typical BEXUS configuration, with a $12000-\mathrm{m}^{3}$ balloon, a valve, a cutter, a parachute, the Esrange Balloon Service System (EBASS), a flight train, Argos GPS, an air traffic control transponder (AGT), a strobe light, a radar reflector, and a gondola. The total length of the system is up to $75 \mathrm{~m} \mathrm{[26].}$

Section IV analyzes the total scattered field. Finally, Section V summarizes the main results of this paper.

\section{EXPERIMENTAL SETUP}

The BEXUS program is implemented under a bilateral agency agreement between the German Aerospace Center (DLR) and the SNSB. The BEXUS 17 stratospheric balloon [see Fig. 1(a) and (b)] launch campaign took place in the Esrange Space Center on October 4-14, 2013. The launch took place on October 10, 2013 at 16:00 (GPS time), and the flight duration was $6 \mathrm{~h}$, with an apogee of $27000 \mathrm{~m}$ (see Fig. 2). The trajectory was a single track (see Fig. 3) from the Esrange Space Center (Sweden) to Juujarvi (Finland).

The experimental setup was composed of the $\mathrm{P}(\mathrm{Y})$ and $\mathrm{C} / \mathrm{A}$ ReflectOmeter (PYCARO) [25], a zenith-looking omnidirectional dual-band (L1, L2) right-hand circular polarized antenna patch to collect the direct GPS signals, a left-hand circular polarized nadir-looking dual-band (L1, L2) antenna array to collect the Earth-reflected signals [see Fig. 4(a) and (b)], three batteries providing up to $100 \mathrm{Wh}$ at low temperatures inside the gondola down to $-40{ }^{\circ} \mathrm{C}$, and an onboard computer for the experiment management. The nadir-looking antenna was composed of two interleaved arrays (L1 and L2) of six elementary antenna patches each. The total gain of the antenna was 13 and

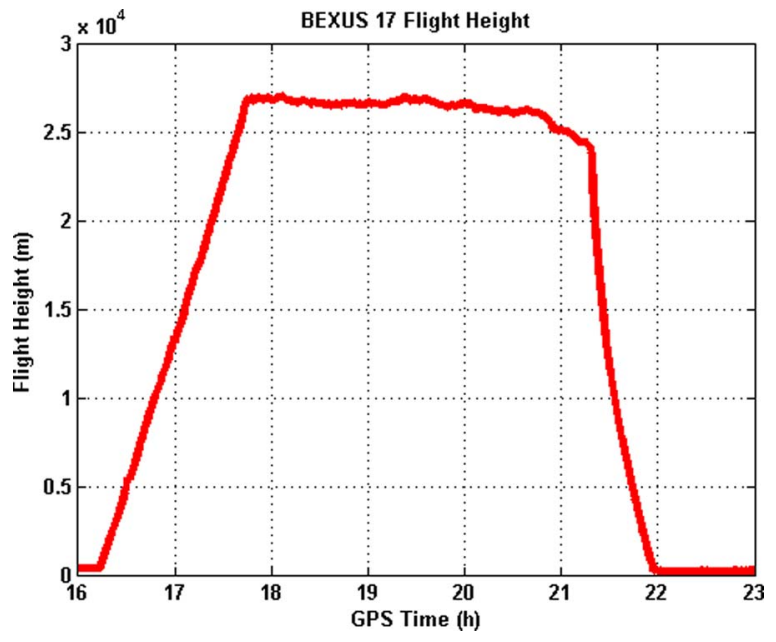

Fig. 2. Flight height during the experiment as a function of the GPS time (October 10, 2013).

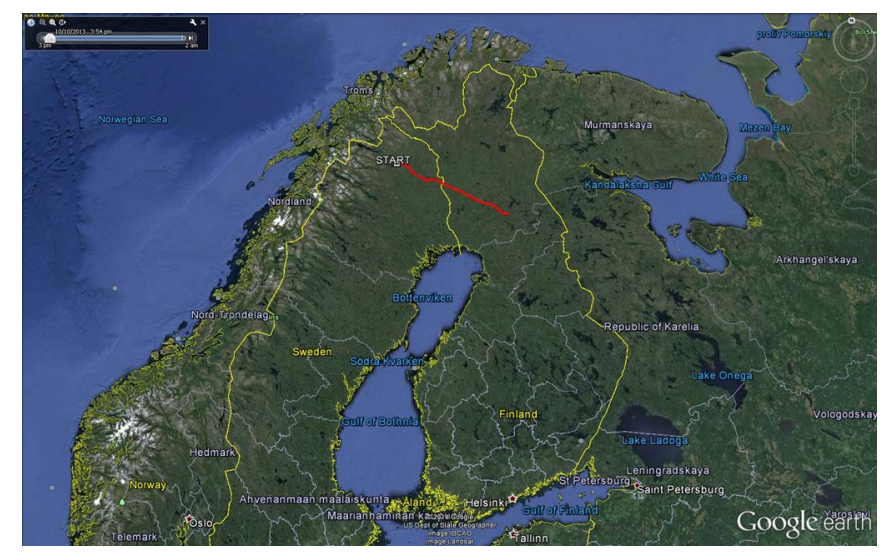

Fig. 3. Track of BEXUS 17 during the experiment. The trajectory was a single track from the Esrange Space Center (latitude $67^{\circ} 53^{\prime} \mathrm{N}$, longitude $21^{\circ} 04^{\prime} \mathrm{E}$ ) to Juujarvi (latitude $66^{\circ} 24^{\prime} \mathrm{N}$, longitude $27^{\circ} 18^{\prime} \mathrm{E}$ ).

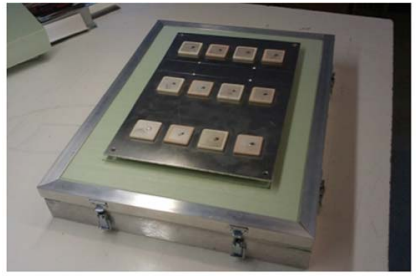

(a)

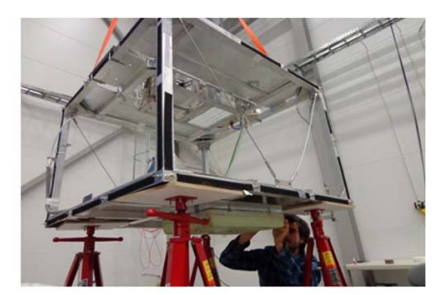

(b)
Fig. 4. (a) Nadir-looking antenna inside the thermally insulating radome. (b) Image of the thermally insulating radome at the bottom of the gondola.

$11 \mathrm{~dB}$ at L1 and L2, respectively. The onboard data handling (OBDH) system was composed of a programmable intelligent computer for housekeeping and scientific data management, communications with the ground station, and data storage in a amicrosecure digital (SD) card. The collected data were registered in two internal SD memory devices (PYCARO and microcontroller), and they were simultaneously sent to the ground segment via the E-Link system [26]. 


\section{EXPERIMENTAL EVALUATION OF THE REFLECTED SIGNALS OVER BOREAL FORESTS}

When an electromagnetic wave impinges from above upon the boundary surface between two semi-infinite media, a portion of the incident energy is scattered toward the upper medium, and the rest is transmitted forward into the lower medium [27, pp. 846]. If the lower medium is homogeneous, the problem in question is a surface scattering problem. On the other hand, if the lower medium is inhomogeneous (i.e., a mixture of materials of different dielectric properties), the scattering takes place within the volume of the lower medium, and it is referred to as volume scattering. Since volume scattering is mainly caused by dielectric discontinuities within a volume and, in general, the spatial locations of discontinuities are random, the scattered waves are expected to be within the volume in all directions. The surface scattering strength is proportional to the relative complex dielectric constant of the lower medium and the surface roughness. The volume scattering strength is proportional to the dielectric discontinuities inside the medium, the density of the embedded discontinuities, the average dielectric constant of the medium, and the geometric size of the inhomogeneities relative to the incident wavelength.

Forests are perfect examples of volume scattering, with scattering elements bounded by the air at the top and by the soil surface at the bottom. The forward scattering coefficient is governed by the scattering properties of the vegetation elements and the soil surface, as well as the interaction between the canopy and the soil, and the soil with the trunks [27, pp. 863], [28]. Several models describing the backscattering of electromagnetic waves over a vegetated surface exist, i.e., from simple threelayer models to models including a continuous medium and a discrete medium characterized by scatterers (e.g., see [29]). However, few studies have been performed to evaluate the forward scattering coefficient at L-band and at circular polarization. In the case of GNSS-R bistatic reflections over a forest area, a scattering model considering both the coherent and incoherent fields was proposed [30]. This model predicts the coherent field as the result of the electromagnetic interactions of the GPS signals with the soil only, which was attenuated by the vegetation canopy above it. Recently, experimental data over forest biomass from 100 to $350 \mathrm{t} / \mathrm{ha}$ have been published [31]. As predicted in [30], a lower value of the coherent soil reflectivity is found for larger vegetation density.

Reflectivity $\Gamma_{r l}^{\mathrm{coh}}$ can be estimated as the ratio of the reflected $Y_{\mathrm{ref}, l}$ and direct $Y_{\mathrm{dir}, r}$ power WF peaks after proper compensation of the noise power floor as follows:

$$
\Gamma_{r l}^{\mathrm{coh}}=\frac{\left|\left\langle Y_{\mathrm{ref}, l}\right\rangle\right|^{2}}{\left|\left\langle Y_{\mathrm{dir}, r}\right\rangle\right|^{2}} .
$$

In (1), subscripts $r$ and $l$ denote the incident polarization (right-hand circular polarization) and the scattered polarization (left-hand circular polarization), respectively.

The scattering of GNSS signals is originated in an area around the nominal specular point. In general, the scattered field contains both a coherent component and an incoherent component in different proportions. The coherent scattering area is limited to the first Fresnel zone. On the other side, the incoherent scattering that is also centered on the nominal specular direction is limited by an area (glistening zone) that is much larger than the first Fresnel zone. The incoherent power component is eliminated in the estimation of the reflectivity in (1) by subtracting from each incoherently averaged WF peak $\left\langle\left|Y_{\mathrm{ref}, l}\right|^{2}\right\rangle$ the amplitude variance of the complex WF peaks $\sigma_{Y_{\text {ref }, l}}^{2}$ as follows [32, pp. 125]:

$$
\left|\left\langle Y_{\text {ref }, l}\right\rangle\right|^{2}=\left\langle\left|Y_{\text {ref }, l}\right|^{2}\right\rangle-\sigma_{Y_{\text {ref }, l}}^{2} .
$$

Therefore, reflectivity values are associated to the first Fresnel zone. The semimajor axis of the first Fresnel zone $\left[r_{a}\right]$ from which the coherent reflections are coming from a flight height of $h=27000 \mathrm{~m}$ and for the $\mathrm{L} 1$ signal is

$$
r_{a}=\sqrt{\lambda h / \sin \theta_{e}+\left(\lambda / 2 \sin \theta_{e}\right)^{2}} / \sin \theta_{e}=78 \mathrm{~m}
$$

where $\lambda$ is the signal's wavelength, and elevation angle $\theta_{e}=$ $70^{\circ}$. The diameter of the antenna footprint varied from 1.4 to $40 \mathrm{~km}$ for a flight height from 1000 to $27000 \mathrm{~m}$, respectively. Since it is much larger than the size of the first Fresnel zone, different reflectivity values were simultaneously measured corresponding to different satellites (different Fresnel zones within the antenna footprint).

In this paper, the bistatic coherent reflectivity $\Gamma_{r l}^{c o h}$ of boreal forests is experimentally evaluated for the first time to the authors' knowledge. When analyzing this problem, it has to be taken into account that the reflected GNSS signal is significantly strong around the specular direction only, and the power density rapidly decreases away from it [30]. In [30], the coherent scattering is assumed to only come from the reflected signals over the soil, which was attenuated by the vegetation canopy. On the other hand, incoherent scattering includes volume scattering from leaves and branches, double bouncing due to the soil and trunk interactions, multiple interactions between vegetation elements and between the vegetation and the soil, and incoherent scattering from the soil attenuated by the overlapping vegetation canopy. However, coherent effects may appear because the distance between scatterers is comparable with the electromagnetic wavelength [27, pp. 827].

The Topography From Reflectometric Measurements: An Experiment From the Stratosphere (TORMES) stratospheric experiment was performed north of Sweden from the Esrange Space Center (latitude $67^{\circ} 53^{\prime} \mathrm{N}$, longitude $21^{\circ} 04^{\prime}$ E) to Juujarvi (latitude $66^{\circ} 24^{\prime} \mathrm{N}$, longitude $27^{\circ} 18^{\prime} \mathrm{E}$ ). The GPS space segment is divided into six orbital planes with an inclination of $\sim 55^{\circ}$. As a consequence, GNSS-R acquisitions were performed with a maximum elevation angle around $75^{\circ}$, and an average of five GPS satellites could be simultaneously tracked during $3 \mathrm{~h}$ each. PYCARO [9], [25] was configured to use the C/A GPS code during this experiment. It measured the direct and reflected WFs using a coherent integration time of $T_{c}=20 \mathrm{~ms}$ and incoherent averaging of $N_{\mathrm{inc}}=10$ samples. These default parameters were implemented in the automatic mode of the OBDH system since, during the experiment preparation activities, it was determined that the SNR increases as a function of the coherent integration time up to $13 \mathrm{~dB}$ using 


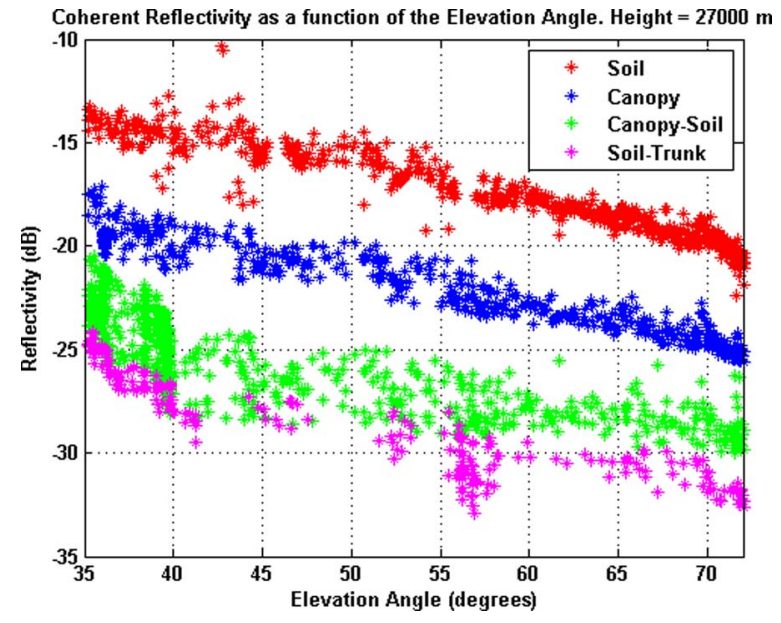

Fig. 5. Evolution of the coherent reflectivity over the different scattering contributing media of the forest (soil, canopy, canopy-soil, and soil-trunk) as a function of the elevation angle at a flight height of $h=27000 \mathrm{~m}$.

$T_{c}=20 \mathrm{~ms}$, as compared with $T_{c}=1 \mathrm{~ms}$. The selection of the number of incoherent averaging samples was a tradeoff between the spatial resolution and the noise filtering. To mitigate the effect of noise, incoherent averaging of consecutive uncorrelated signals must be performed. However, the larger the number of incoherently averaged complex WFs, the lower the spatial resolution. In this experiment, the spatial resolution was very slightly degraded because of the low speed of the balloon. Unfortunately, just after takeoff, the BEXUS E-Link failed, and the experiment was autonomously operated during the whole flight. As a first step, the 20-ms coherently integrated WFs were computed onboard. The reflected GPS signals were multiplied by a locally generated carrier signal and with a $90^{\circ}$ phase-shifted signal to generate the inphase and quadrature components, respectively. Then, the Fourier transform of the complex input signal was multiplied with the Fourier transform of the transmitted pseudorandom noise (PRN) code. The result of the multiplication was transformed into the time domain by an inverse Fourier transform. The WFs were properly aligned to compensate for the GPS satellites' motion [33]. Then, they were incoherently averaged $\left(N_{\text {inc }}=10\right)$ to reduce the effect of the noise.

PYCARO [9], [25] was configured to track the correlation peak of each temporal measurement during this experiment. After the flight, during data processing, the reflection coefficients were computed by applying (1) to the temporal series of data provided by the reflectometer. The temporal evolution of the coherent reflectivity at a flight height of $h=27000 \mathrm{~m}$ is represented in Fig. 5 as a function of elevation angle $\theta_{e}$ after compensating for the antenna pattern gain. It can be observed that, as expected, the higher the elevation angles (closer to zenith), the lower the values of coherent reflectivity [27, pp.1008].

Boreal forests are characterized by coniferous forests consisting mostly of pines, spruces, and larches. Boreal forests can be described using allometric relationships [34]. In the following, ground-truth data and data provided by airborne Lidar are included as additional information in this paper [34]. In Fig. 6(a), height versus biomass from ground measurement
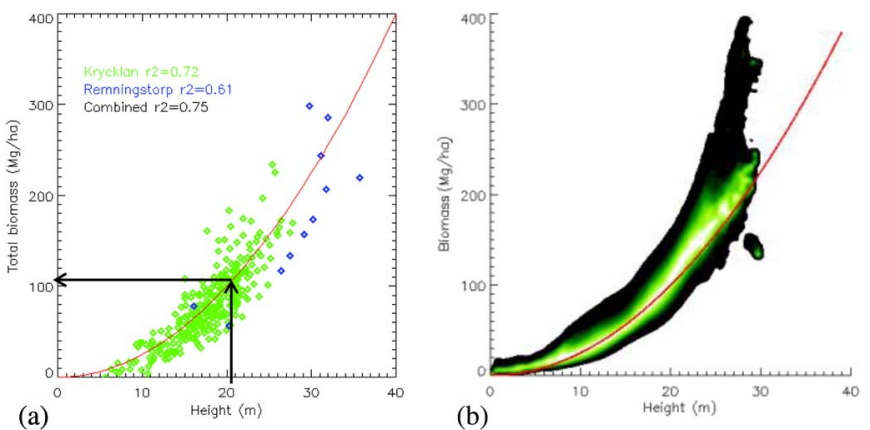

Fig. 6. (a) Ground-truth plots in Krycklan (green) and Remningstorp (blue). (b) Krycklan site for airborne Lidar height versus airborne Lidar-derived biomass (adapted from the work in [34]).

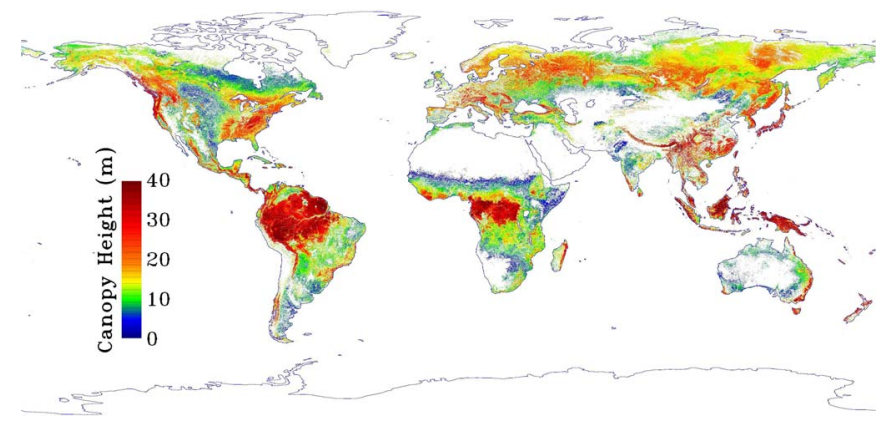

Fig. 7. Global map of the forest height produced from NASA's Ice, Cloud, and Land Elevation Satellite (ICESat)/Geoscience Laser Altimeter System, Moderate Resolution Imaging Spectroradiometer (MODIS), and Tropical Rainfall Measuring Mission sensors [35].

plots is shown. The measurements for the two test sites, i.e., Krycklan (latitude $64^{\circ} 10^{\prime} \mathrm{N}$, longitude $20^{\circ} 01^{\prime} \mathrm{E}$ ) in green and Remningstorp (latitude $58^{\circ} 25^{\prime} \mathrm{N}$, longitude $13^{\circ} 14^{\prime} \mathrm{E}$ ) in blue, show a high correlation $\left(R^{2}=0.75\right)$, and both follow the same allometric relationships as follows:

$$
P=0.25 h_{\text {forest }}^{2}
$$

where $P$ is the biomass (in tons per hectare), and $h_{\text {forest }}$ is the forest height (in meters). Fig. 6(b) shows the height-to-biomass relationship derived from airborne Lidar data for the Krycklan test site. It follows the same allometric relationship as for the ground measurements. Using the data provided by NASA (see Fig. 7), an estimation of the biomass for Krycklan using (4) is $P \sim 100$ t/ha.

The multimodal behavior shown in Fig. 5 suggests that the coherent scattering may take place in differentiated scattering media with different scattering properties. The fluctuations of the coherent reflectivity as derived from the peak of the WF follow four different trends, each with different levels and relative variations of the reflectivity. Boreal forests are characterized by random variations of the dielectric properties. Different scattering media $^{2}$ (soil, trunks, branches, and leaves), each with particular dielectric properties, may lead to different reflected power

\footnotetext{
${ }^{2}$ The terrain type on the flight path was inspected by the authors during a car travel. Fig. 8 shows four shots of the main scattering-type scenarios found in the flight path. These four scenarios alternatively appear on the path.
} 


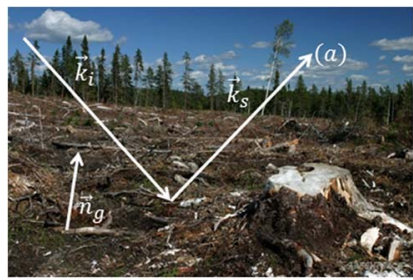

(a)

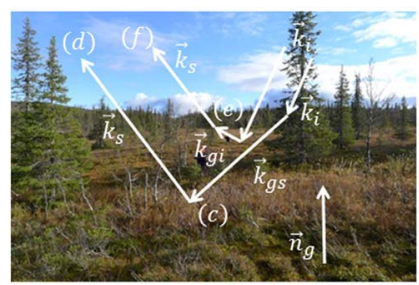

(c)

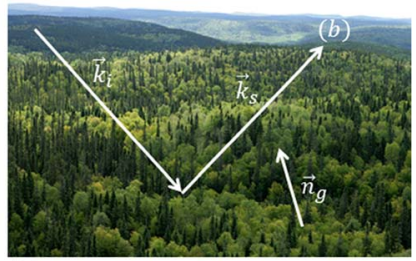

(b)

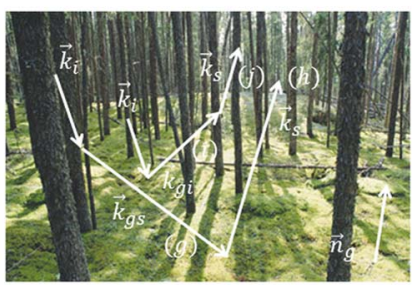

(d)

Fig. 8. (a) Scattering over the ground surface. (b) Direct scattering over the canopy. (c) Multiple scattering involving both the soil and the canopy. (d) Multiple scattering involving both the soil and the trunks.

levels of the signals. As a consequence of the small size of the coherent scattering area (limited by the first Fresnel zone), the different dielectric properties of the forest elements, the different levels of signal attenuation due to multiple reflections and the different heights of the scatterers, and the suggestion by $\mathrm{Wu}$ and Jin [28] that the forward scattering of GNSS signals takes place not only over the soil but also over trees in a multimodal behavior, we hypothesize that the different scattering mechanisms are sequentially dominating (see Fig. 5) over the soil (red), the canopy (blue), and the canopy-soil (green) and soil-trunk (pink) interactions. This result indicates that coherent scattering is also taking place in the canopy and trunks. Depending on the vegetation cover fraction, the scattered power may be composed of several contributions. Fig. 8(a) and (b) shows direct scattering from the soil and the canopy, respectively, and Fig. 8(c) and (d) shows multiple reflected scattering involving both the soil and the canopy, and both trunks and the soil, respectively.

Each incoherently averaged WF was composed of several correlation peaks. PYCARO was configured to track the highest peak of each WF. The scattering over the canopy in a multimodal behavior produces reflected signals with different delays. Additionally, note that the effect of the topography can produce coherent scattering (under a specular condition) from areas (several first Fresnel zones) that do not correspond to the smaller geometric delay. Thus, only the scattering mechanism with the highest power contribution to the total scattered field can be identified at each individual WF $\left(T_{c}=20 \mathrm{~ms}\right.$ and $N_{\text {inc }}=10$ ). In addition, the histogram corresponding to the total scattered field over boreal forests at a flight height of $h=27000 \mathrm{~m}$ and for high elevation angles in the range of $\theta_{e}=\left[35^{\circ}, 72^{\circ}\right]$ is represented in Fig. 9, where four amplitude distributions can be identified depending on the level of coherence of the reflected signal during the flight. These distributions are obtained taking into account the four different types of scattering mechanisms (soil, canopy, canopy-soil, and soil-trunk) considered in this section.

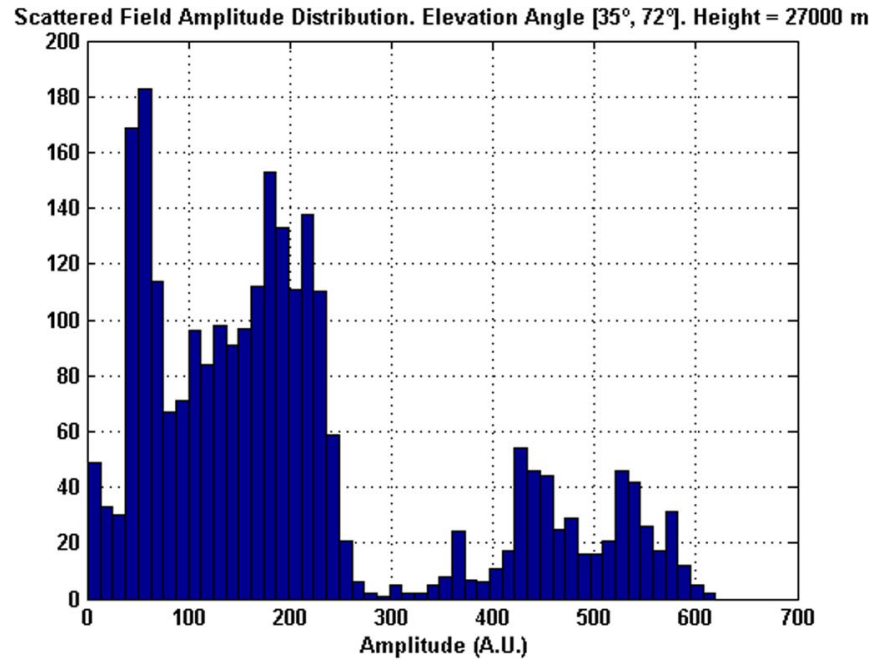

Fig. 9. Total scattered field (over boreal forests) amplitude distribution histogram at a flight height of $h=27000 \mathrm{~m}$ and for an elevation angle in the range of $\theta_{e}=\left[35^{\circ}, 72^{\circ}\right]$. There are four main peaks (larger number of samples at these four identified amplitude levels), i.e., $\sim 50, \sim 190, \sim 420$, and $\sim 520$ (A.U.).

The amount of coherent reflected power $P_{r l}^{\text {coh }}$ can be obtained by applying (5) with the antennas separated by a distance $R=R_{t 0}+R_{0 \mathrm{rc}}$ as follows [19], [36]:

$$
P_{r l}^{\mathrm{coh}}=\Gamma_{r l}^{\mathrm{coh}} \frac{P_{t} \lambda^{2} G_{t} G_{\mathrm{rc}}}{(4 \pi)^{2}\left(R_{t 0}+R_{0 \mathrm{rc}}\right)^{2}}
$$

where $P_{t}$ is the power emitted by the GPS satellites, $G_{t}$ is the gain of the transmitting antenna, and $G_{\mathrm{rc}}$ is the gain of the receiving antenna. Since $R_{t 0} \gg R_{0 \mathrm{rc}}$, the reflected coherent power is approximately constant, i.e.,

$$
P_{r l}^{\mathrm{coh}} \approx \Gamma_{r l}^{\mathrm{coh}} \frac{P_{t} \lambda^{2} G_{t} G_{\mathrm{rc}}}{(4 \pi)^{2}\left(R_{t 0}\right)^{2}} .
$$

On the other hand, the incoherent power can be theoretically described as [17]

$$
P_{r l}^{\text {incoh }}=\frac{P_{t} \lambda^{2} T_{c}^{2}}{(4 \pi)^{3}} \quad \frac{G_{t} G_{\mathrm{rc}} \Lambda^{2}(\delta \tau) S^{2}(\delta f)}{\left(R_{t 0}\right)^{2}\left(R_{0 \mathrm{rc}}\right)^{2}} \sigma_{r l}^{0} d A
$$

where $\Lambda$ is the autocorrelation function of the GNSS code; $S$ is the sinc exponential function; $\delta \tau$ and $\delta f$ are the differences between sampled time delay $\tau$ and sampled frequency $f$ and a reference delay and a Doppler frequency, respectively; $\sigma_{r l}^{0}$ is the incoherent bistatic radar scattering coefficient, and $A$ is the integration area. The scattered signal may be Doppler limited if the Doppler filter corresponding to a high coherent integration time up to $T_{c}=20 \mathrm{~m}$ is smaller than the first C/A chip size. An analysis has been performed for different elevation angles in the range of $\theta_{e}=\left[45^{\circ}, 75^{\circ}\right]$ and for different platform heights in the range of $h=[1000,27000] \mathrm{m}$ using the Passive Advanced Unit (PAU)/PARIS End-to-End Performance Simulator (PEPS) [37]. Results show that there are only Doppler bandwidth effects for a flight height $h \sim 1000 \mathrm{~m}$ [see Fig. 10(a) and (b)]. For the flight conditions $(h \sim 27000 \mathrm{~m}$ and scattering over land surfaces), the Earth region contributing to the incoherent component is the first chip isorange ellipse that is a function of the autocorrelation function of the different GNSS codes. 


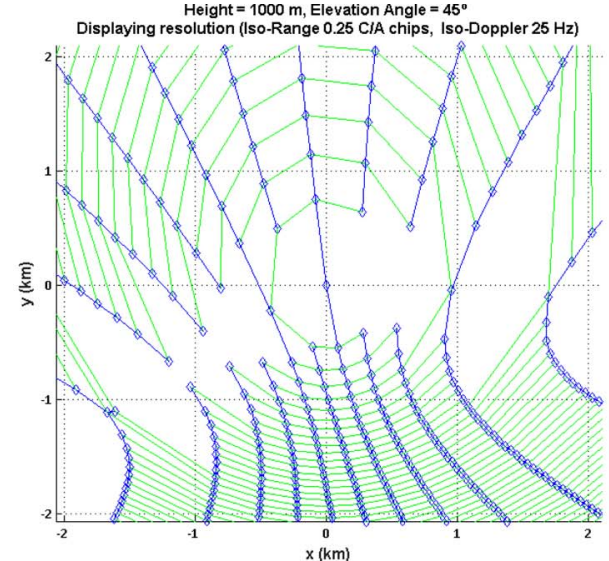

(a)

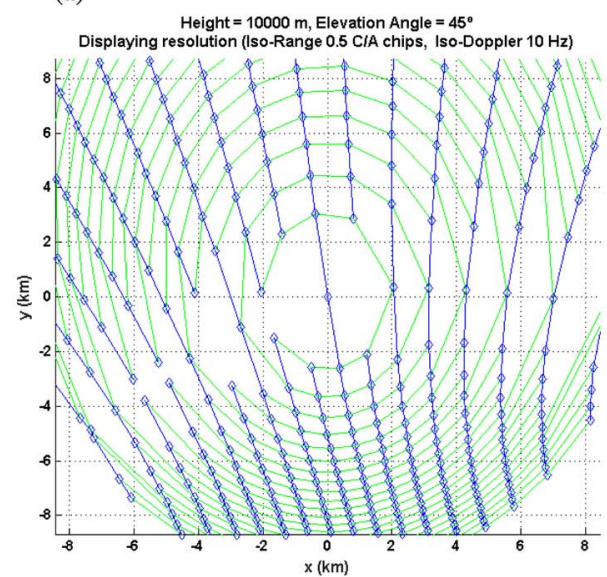

(c)

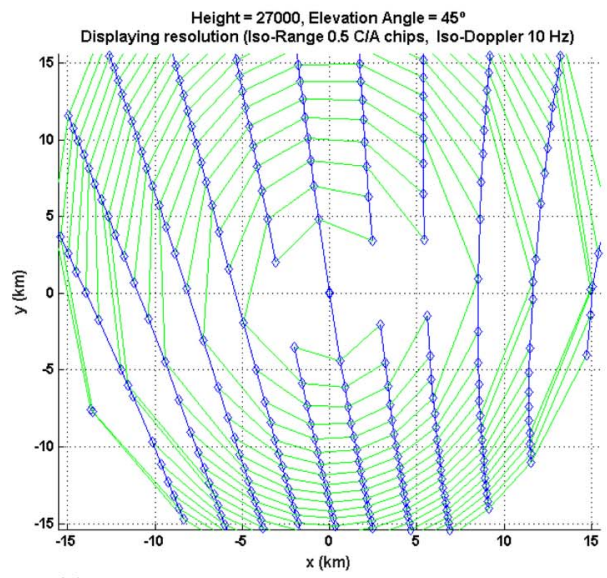

(e)

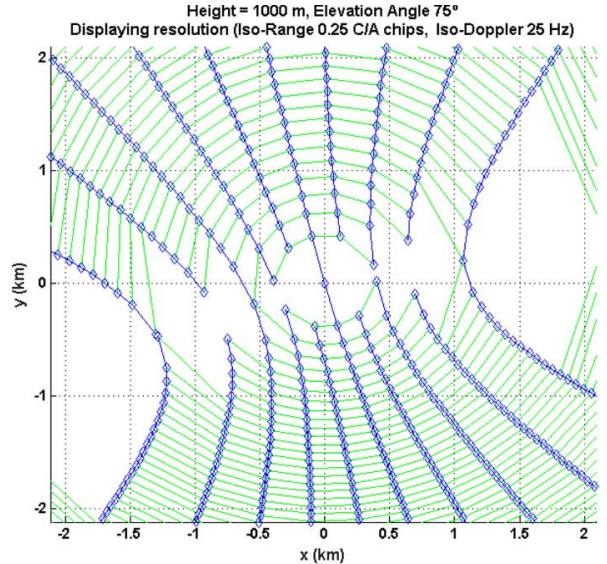

(b)

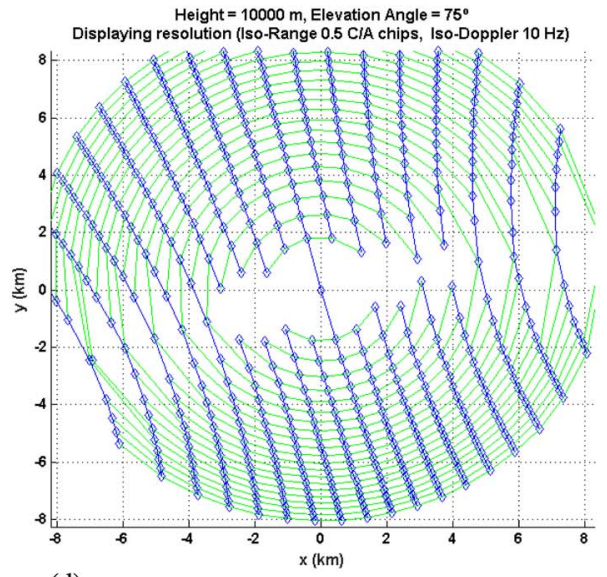

(d)

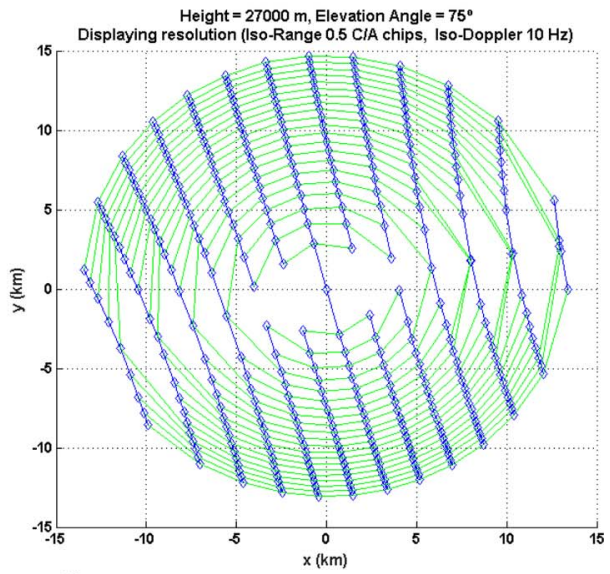

(f)

Fig. 10. Isorange (green lines) and iso-Doppler (blue lines) lines for different flight conditions. (a) $h=1000 \mathrm{~m}$ and $\theta_{e}=45^{\circ}$. (b) $h=1000 \mathrm{~m}$ and $\theta_{e}=75^{\circ}$. (c) $h=10000 \mathrm{~m}$ and $\theta_{e}=45^{\circ}$. (d) $h=10000 \mathrm{~m}$ and $\theta_{e}=75^{\circ}$. (e) $h=27000 \mathrm{~m}$ and $\theta_{e}=45^{\circ}$. (f) $h=27000 \mathrm{~m}$ and $\theta_{e}=75^{\circ}$.

However, in the case of ocean scattering, the spreading of the signal over the surface includes many chips. The area of the first isorange ellipse is equal to [38]

$$
A=\pi a b=\frac{2 c \tau \pi R_{0 \mathrm{rc}}}{\left(\sin \theta_{e}\right)^{2}}
$$

where $a$ and $b$ are the semimajor and semiminor axes of the first isodelay ellipse, respectively, $c$ is the speed of light, and $\tau$ is the chip size of the PRN [38]. Therefore, the incoherent reflected power is proportional to $\sim 1 / R_{0 \mathrm{rc}}$ versus the coherent scat- tering that can be theoretically modeled independent of $R_{0 \mathrm{rc}}$ [see (6)]. Fig. 11 shows that the evolution of the reflected power as a function of the flight height in the range from $h=0$ to $h=$ $20000 \mathrm{~m}$ is roughly constant for the range of heights involved since the coherent integration time was set to be $T_{c}=20 \mathrm{~ms}$ to limit the incoherent scattering.

\section{ANAlysis of the Total Scattered Field}

In Section II, the reflectivity was analyzed considering the temporal evolution of the scattered signals, finding out just from 


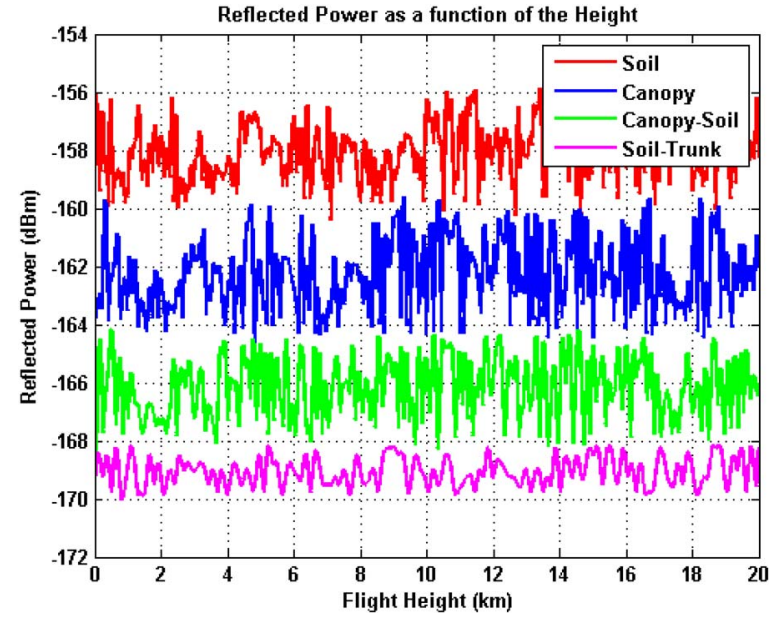

Fig. 11. Reflected power evolution as a function of the flight height and the scattering media.

power considerations that there was a strong coherent component. Now, using the inphase and quadrature components, the total scattered field is analyzed. The total scattered field of this configuration of scatterers $(\sim 15$-min-long data set each over boreal forests) can be described as a vector sum in the complex plane of the temporal measurements provided by PYCARO. The coherent ${ }^{3}$ versus incoherent scattering analysis is performed using the complete data sets of 15 min each. Instantaneously, during each coherent integration time, the reflected signals are highly coherent [9], [25], and PYCARO was able to track the phase (of the coherent component) of the reflected signal because the coherent integration time was set to be very high, i.e., $T_{c}=20 \mathrm{~ms}$, to limit the incoherent scattering. However, when the complete temporal series of data is taken into account, the resulting reflected signal (the sum of the instantaneous signal vectors) is composed of both coherent and incoherent components. The contribution of the four different types of scattering mechanisms (soil, canopy, canopy-soil, and soil-trunk) to the total scattered field is equally distributed in the same region of the complex plane [see Fig. 12]. Fig. 12(a)-(c) shows the total scattered complex field distribution for three different flight height ranges $h=[0,5000] \mathrm{m}, h=[20000,25000] \mathrm{m}$, and $h \sim 27000 \mathrm{~m}$ for midlow elevation angles, respectively, and Fig. 12(d)-(f) shows that for high elevation angles. If the scattered complex field described a circle centered around $(0,0)$, the scattering ${ }^{4}$ would be completely incoherent. However, the scattered field is displaced from the origin ${ }^{5}$ by a value $\alpha$ (equal to the mean of the amplitude distribution). The relative weight of the coherent to the incoherent components is quantified by the following parameter [32, pp. 126]:

$$
B^{2}=\frac{\alpha^{2}}{s_{1}+s_{2}}
$$

\footnotetext{
${ }^{3}$ Valencia et al. [39] show the existence of a coherent component in addition to the incoherent component.

${ }^{4}$ In a general case, the resulting scattered field can be described as the sum of a constant vector and a Hoyt vector that is defined as a 2-D Gaussian distribution with mean zero value and variances $s_{1}$ and $s_{2}$ [32, pp. 125].

${ }^{5}$ There are two regions displaced by $\pm \alpha$ from the center because the phase changes due to the navigation bit changing sign.
}

where $\alpha$ is the mean amplitude distribution, and $s_{1}$ and $s_{2}$ are the variances of the inphase and quadrature components of the scattered field, respectively (see Table I). Note that $B$ tends to $\infty$ for a totally coherent field, and it is equal to 0 for a totally incoherent field. The evolution of this parameter for each type of scattering element is represented as a function of the flight height in Fig. 13(a) for high elevation angles in the range of $\theta_{e}=\left[60^{\circ}, 70^{\circ}\right]$ and in Fig. 13(b) for low elevation angles in the range of $\theta_{e}=\left[25^{\circ}, 45^{\circ}\right]$, showing that the relative weight is roughly independent of the flight height for a reconstructed scattered field corresponding to an along track ${ }^{6}$ of $\sim 25 \mathrm{~km}$.

The coherent and incoherent components of the reconstructed field ( $25 \mathrm{~km}$ along track) tend to increase with the flight height (see Table I) because of the larger noise of the received signals during the takeoff (see Fig. 12) due to the attitude oscillations of the gondola. At a flight height in the range of $h=$ $[20000,25000] \mathrm{m}$, the scattering area is larger (see Table I) that partially mitigates these oscillations; however, also note that, during this part of the flight, the trajectory was being totally stable during the float phase with an apogee of $h \sim 27000 \mathrm{~m}$. Note that this effect ${ }^{7}$ is only visible in the reconstructed field because, during the coherent integration time, the orientation of the gondola is practically frozen (see Section II). In Fig. 12, it is clear that the clusters of points are better defined in Fig. 12(c) and (f) than in Fig. 12(a) and (d). This behavior is evaluated using the asymmetry factor defined as

$$
K=\frac{s_{1}}{s_{2}}
$$

It is observed that the asymmetry factor of each forest contribution increases with the flight height, with the asymmetry factor being larger in the case of low elevation angles (see Table I). It means that the phase is less noisier at a flight height of $h=[20000,25000] \mathrm{m}$ than in the range of $h=[0,5000] \mathrm{m}$, which explains the clustered behavior [see Fig. 12(c) and (f)]. In particular, the asymmetry factor increment from the ascend to the float phase is in the range of $K=[15,35]$ A.U. for lower elevation angles, whereas for high elevation angles, it is in the range of $K=[12,18]$ A.U. (see Table I). The phase information is retrieved from the coherent component of the scattered field. Additionally, it can be stated that the effect of the amplitude noise is larger than the effect of the phase noise since the asymmetry factor is larger than 1 in all cases. The multimodal behavior due to the scattering over different types of scatterers creates fluctuations in the amplitude of the signal, which is larger than the phase noise.

The distributions of the reflected WF peak phase $\left(T_{c}=\right.$ $20 \mathrm{~ms}$, the number of incoherent averaging samples $N_{\text {inc }}=10$ ) as measured by PYCARO before the GPS signal is retracked are represented in Fig. 14(a) and (b) for high and midlow elevation angles, respectively, and for a flight height of $h \sim 27000 \mathrm{~m}$. These distributions are fitted by log-logistic probability density

\footnotetext{
${ }^{6}$ The horizontal speed of the balloon was approximately $100 \mathrm{~km} / \mathrm{h}$, and the duration of the data sets is 15 min each.

${ }^{7}$ The flight data provided by the Swedish Space Corporation were computed onboard the balloon. The vertical speed of the balloon during the float phase was smaller than $1 \mathrm{~m} / \mathrm{s}$.
} 
Scattered Field (Complex Plane). Elevation Angle $\left[24^{\circ}, 3^{\circ}\right]$. Height $[0 \mathrm{~m}, 5000 \mathrm{~m}]$

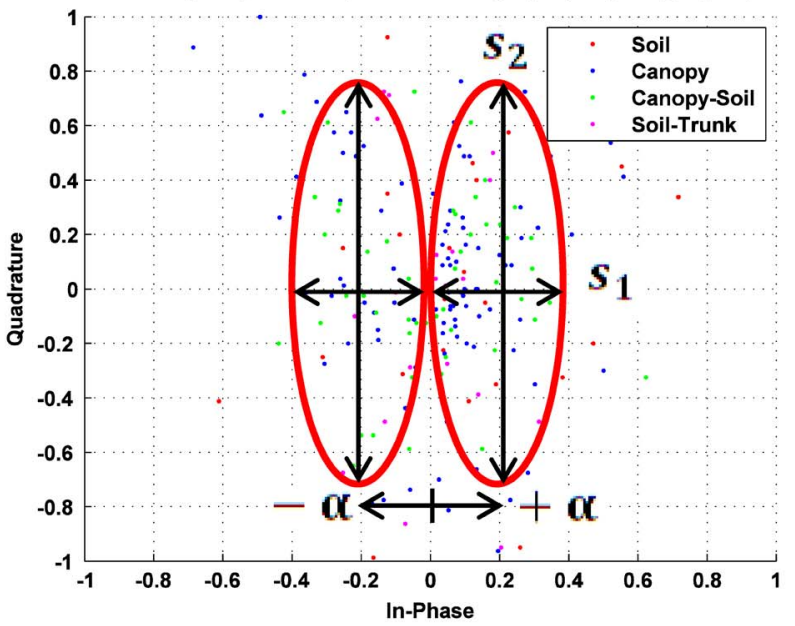

(a)

Scattered Field (Complex Plane): Elevation Angle [21 $\left.{ }^{\circ}, 3^{\circ}\right]$. Height $[20000 \mathrm{~m}, 25000 \mathrm{~m}$

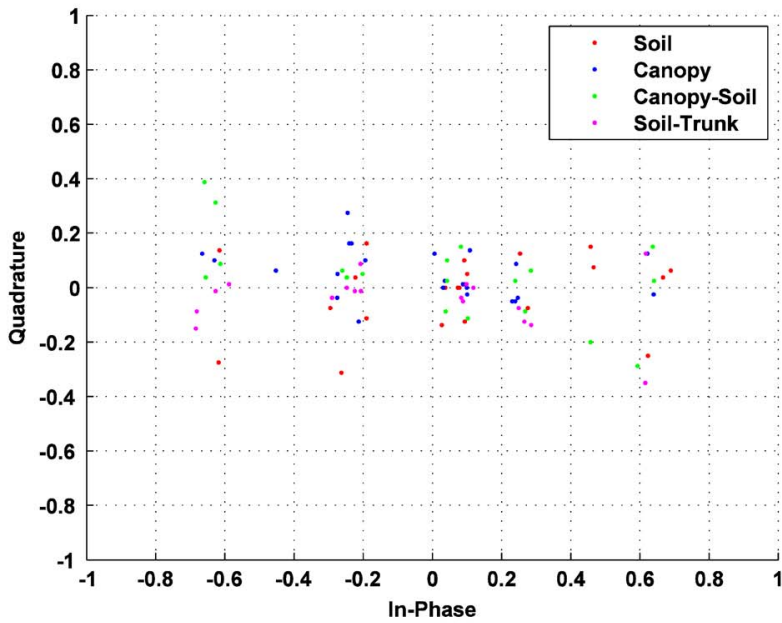

(b)

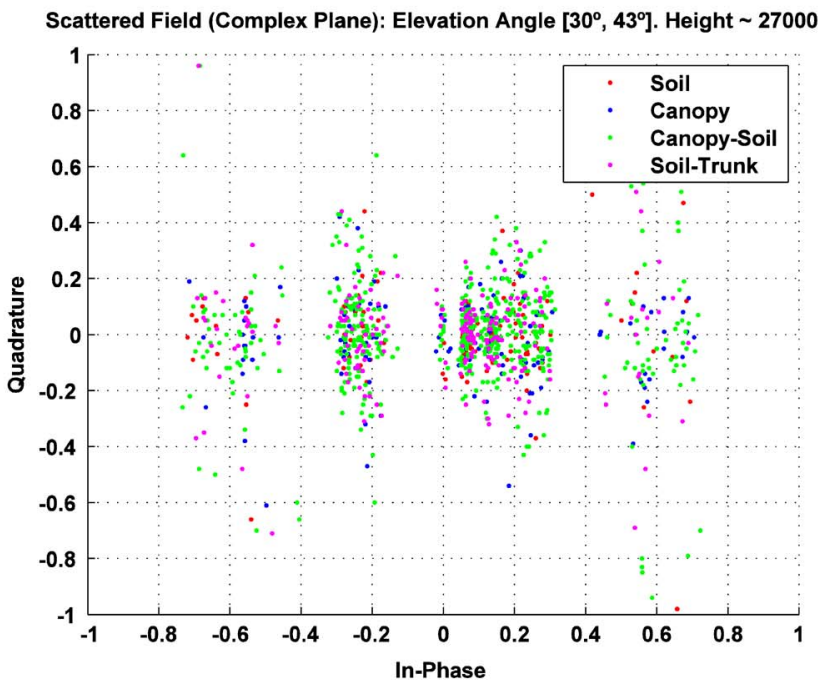

(c)

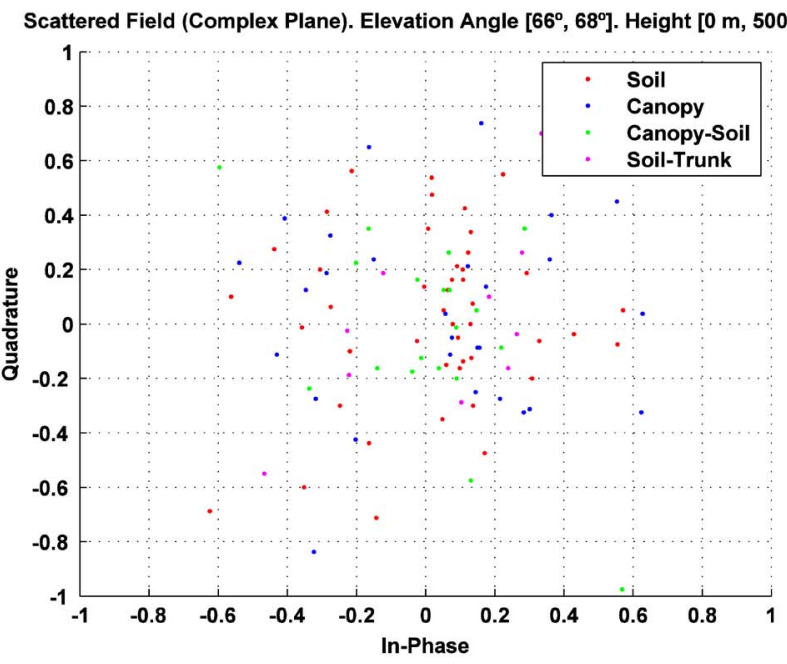

(d)

Scattered Field (Complex Plane). Elevation Angle [65 $\left.{ }^{\circ}, 7^{\circ}\right]$. Height $[20000 \mathrm{~m}, 25000 \mathrm{~m}]$

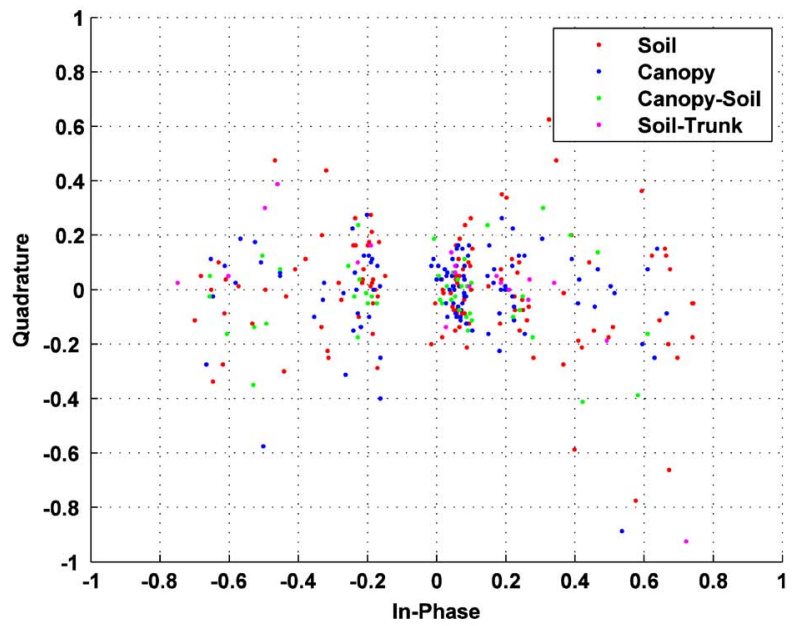

(e)

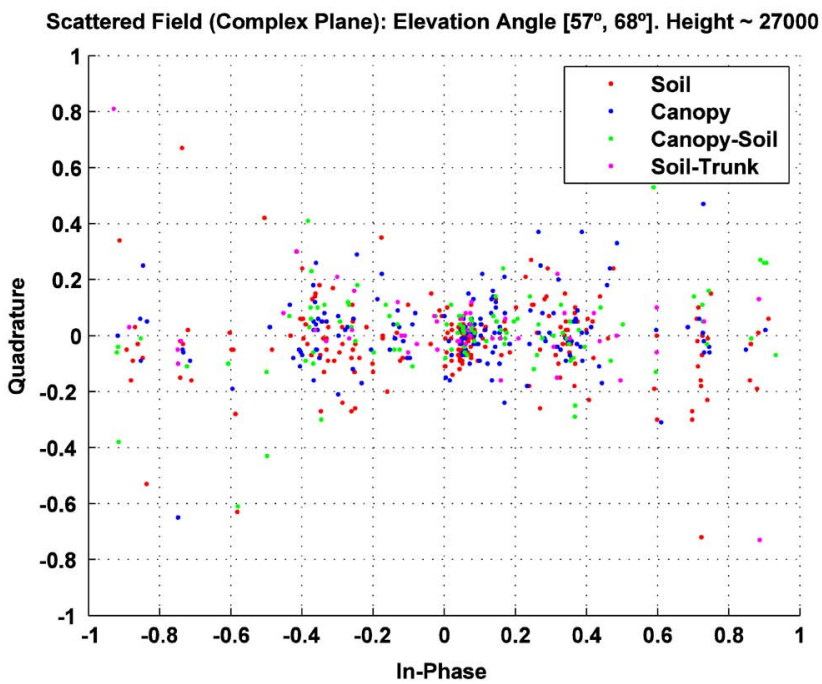

(f)

Fig. 12. (a) Scattered field complex plane representation for a flight height $h=[0,5000] \mathrm{m}$ and an elevation angle in the range of $\theta_{e}=\left[24^{\circ}, 35^{\circ}\right]$. (b) Scattered field complex plane representation for a flight height $h=[20000,25000] \mathrm{m}$ and an elevation angle in the range of $\theta_{e}=\left[21^{\circ}, 30^{\circ}\right]$. (c) Scattered field complex plane representation for a flight height $h \sim 27000 \mathrm{~m}$ and an elevation angle in the range of $\theta_{e}=\left[30^{\circ}, 43^{\circ}\right.$. (d) Scattered field complex plane representation for a flight height $h=[0,5000] \mathrm{m}$ and an elevation angle in the range of $\theta_{e}=\left[66^{\circ}, 68^{\circ}\right]$. (e) Scattered field complex plane representation for a flight height $h=[20000,25000] \mathrm{m}$ and an elevation angle in the range of $\theta_{e}=\left[65^{\circ}, 71^{\circ}\right]$. (f) Scattered field complex plane representation for a flight height $h \sim 27000 \mathrm{~m}$ and an elevation angle in the range of $\theta_{e}=\left[57^{\circ}, 68^{\circ}\right]$. Note: The normalization factor of each axis (inphase and quadrature) of each figure [from (a) to (f)] is the maximum value. 

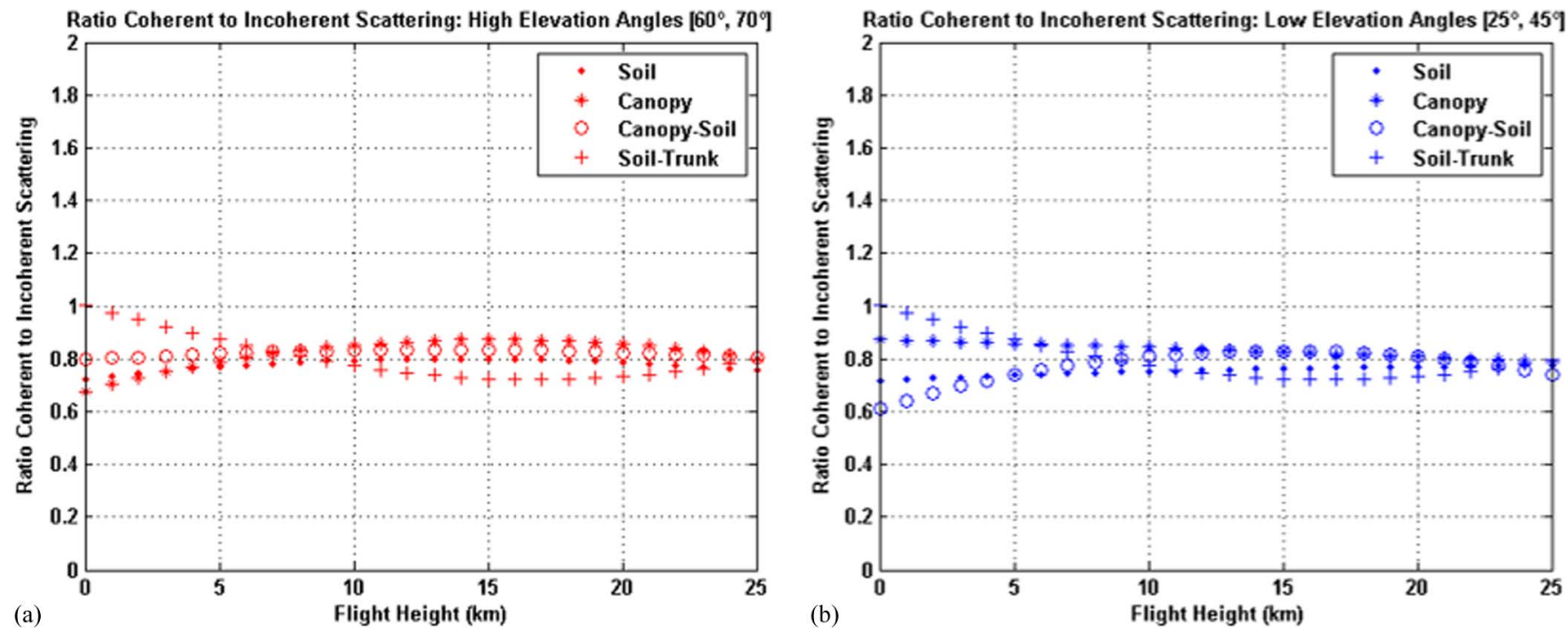

Fig. 13. (a) Evolution of the ratio of the coherent to incoherent scattering as a function of the flight height and for an elevation angle in the range of $\theta_{e}=$ $\left[60^{\circ}, 70^{\circ}\right]$. (b) Evolution of the weight of the coherent to incoherent scattering as a function of the flight height and for an elevation angle in the range of $\theta_{e}=\left[25^{\circ}, 45^{\circ}\right]$.

TABLE I

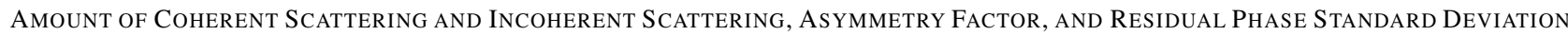
Over Soll, CANopy, CANOpy-Soil, and SoIl-Trunk as a Function of the Flight Height and the Elevation Angle

\begin{tabular}{|c|c|c|c|c|}
\hline & $\begin{array}{c}\text { Cluster } 1 \\
\text { (red) }\end{array}$ & $\begin{array}{c}\text { Cluster } 2 \\
\text { (blue) }\end{array}$ & $\begin{array}{c}\text { Cluster } 3 \\
\text { (green) }\end{array}$ & $\begin{array}{c}\text { Cluster } 4 \\
\text { (pink) }\end{array}$ \\
\hline \multicolumn{5}{|l|}{$h=[0,5,000] \mathrm{m}, \theta_{e}=\left[65^{\circ}, 70^{\circ}\right]$} \\
\hline Coherent Scattering: $\alpha^{2}$ (A.U.) & 32,761 & 54,756 & 20,449 & 44,944 \\
\hline Incoherent Scattering: $s_{1}+s_{2}$ (A.U.) & 51,650 & 73,021 & 37,837 & 58,642 \\
\hline Asymmetry Factor: $K=s_{1} / s_{2}$ & 4.1 & 5.3 & 4.1 & 6.1 \\
\hline Residual Phase Std. (degrees) & 24.7 & 14.4 & 22 & 8.8 \\
\hline \multicolumn{5}{|l|}{$h=[0,5,000] \mathrm{m}, \theta_{e}=\left[30^{\circ}, 35^{\circ}\right]$} \\
\hline Coherent Scattering: $\alpha^{2}$ (A.U.) & 43,264 & 33,124 & 29,929 & 49,729 \\
\hline Incoherent Scattering: $s_{1}+s_{2}$ (A.U.) & 73,021 & 52,432 & 44,234 & 81,917 \\
\hline Asymmetry Factor: $K=s_{1} / s_{2}$ & 3.7 & 2.8 & 4.4 & 26 \\
\hline Residual Phase Std. (degrees) & 18.7 & 25.5 & 18.9 & 2.8 \\
\hline \multicolumn{5}{|l|}{$h=[20,000,25,000] \mathrm{m}, \theta_{e}=\left[65^{\circ}, 70^{\circ}\right]$} \\
\hline Coherent Scattering: $\alpha^{2}$ (A.U.) & 34,969 & 41,616 & 33,856 & 29,929 \\
\hline Incoherent Scattering: $s_{1}+s_{2}$ (A.U.) & 56,813 & 67,796 & 58,685 & 46,369 \\
\hline Asymmetry Factor: $K=s_{1} / s_{2}$ & 18.3 & 18.5 & 22 & 17.9 \\
\hline Residual Phase Std. (degrees) & 9 & 12.4 & 15.7 & 8.4 \\
\hline \multicolumn{5}{|l|}{$h=[20,000,25,000] \mathrm{m}, \theta_{e}=\left[30^{\circ}, 35^{\circ}\right]$} \\
\hline Coherent Scattering: $\alpha^{2}$ (A.U.) & 54,756 & 45,639 & 33,124 & 75,076 \\
\hline Incoherent Scattering: $s_{1}+s_{2}$ (A.U.) & 86,557 & 72,410 & 121,248 & 107,648 \\
\hline Asymmetry Factor: $K=s_{1} / s_{2}$ & 26.7 & 38.4 & 29 & 41 \\
\hline Residual Phase Std. (degrees) & 6.6 & 13 & 5.4 & 1.6 \\
\hline
\end{tabular}

functions (pdfs). It has been reported that, in a foliage environment, log-logistic pdfs can provide more accurate fitting of the amplitude of multipath impulse responses other than lognormal, Weibull, and Rayleigh models for narrowband signals [40]. The Kolmogorov-Smirnov test was used to perform a goodness-of-fit test. This test rejected the null hypothesis that the phase comes from Weibull, Rayleigh, and log-logistic distributions at $9 \%, 6 \%$, and $3 \%$ of the significance level, respectively. The log-logistic distribution for this particular set of data is the more accurate distribution that is in agreement with the work in [40]. The maximum likelihood estimates for the parameters are mean $\mu=2.47^{\circ}$ and scale parameter $\beta=1.48$ 
Phase Standard Deviation Distribution. Elevation Angle $\left[58^{\circ}, 70^{\circ}\right]$. Height $=27000 \mathrm{~m}$

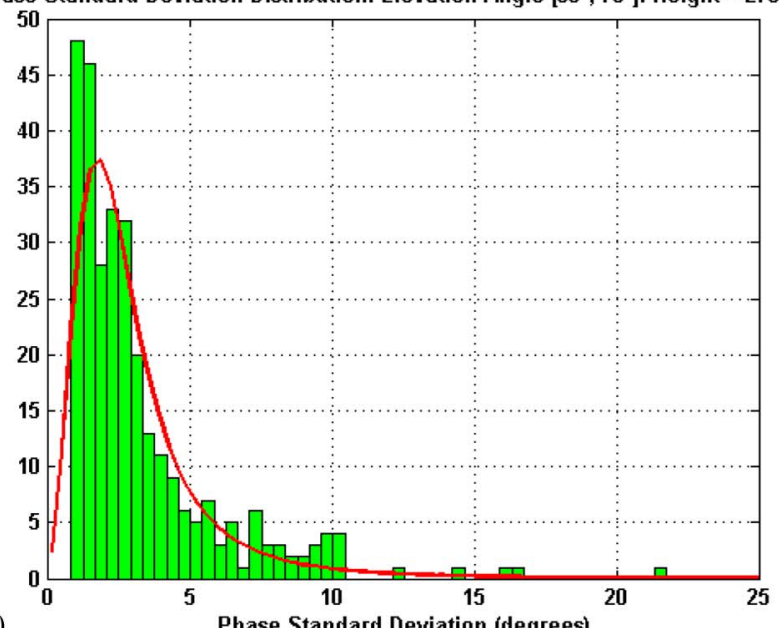

(a)

Phase Standard Deviation (degrees)
Phase Standard Deviation Distribution. Elevation Angle $\left[30^{\circ}, 40^{\circ}\right.$. Height $=27000 \mathrm{~m}$

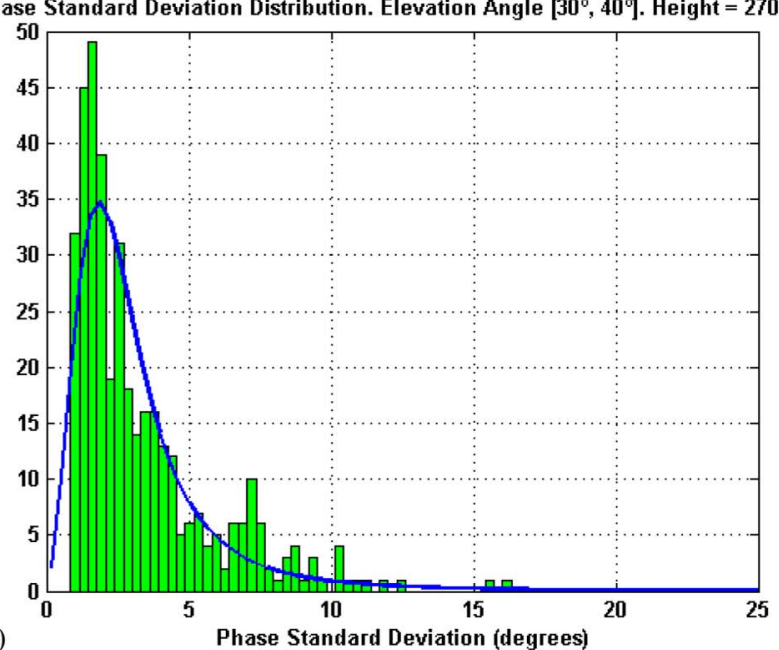

Fig. 14. (a) Carrier phase standard deviation distribution for a flight height of $h \sim 27000 \mathrm{~m}$ and an elevation angle $\theta_{e}=\left[58^{\circ}, 70^{\circ}\right]$. (b) Carrier phase standard deviation distribution for a flight height of $h \sim 27000 \mathrm{~m}$ and an elevation angle $\theta_{e}=\left[30^{\circ}, 40^{\circ}\right]$.

for high $\left[58^{\circ}, 70^{\circ}\right]$ elevation angles and mean $\mu=2.57^{\circ}$ and scale parameter $\beta=1.48$ for low $\left[30^{\circ}, 40^{\circ}\right.$ ] elevation angles.

Increasing the flight height reduces the phase noise (see Table I) of consecutive samples after signal retracking. The retracking strategy implemented in PYCARO tends to align the sum of the inphase and quadrature components of the scattered field with the inphase axis, and it switches $180^{\circ}$ during each data bit reversal. The purpose of this retracking strategy was to properly align the WFs before the incoherent averaging was performed [33]. The coherently integrated WFs need to be aligned to compensate for the GPS satellites' motion. This motion induces a change in the delay difference of the direct and reflected WFs during the incoherent averaging. The performance of a spaceborne GNSS-R altimeter is seriously degraded without the alignment of the WF samples [33]. Thus, Figs. 12 and 13 deal with the phase after demodulation as provided by the lock-loop mechanism implemented to perform the retracking of the WFs.

\section{Summary AND CONCLUSION}

This paper has described, to the authors' knowledge, the firstever GNSS-R stratospheric experiment over land surfaces. The BEXUS 17 stratospheric flight trajectory was a single track from the Esrange Space Center (latitude $67^{\circ} 53^{\prime} \mathrm{N}$, longitude $21^{\circ} 04^{\prime} \mathrm{E}$ ) to Juujarvi (latitude $66^{\circ} 24^{\prime} \mathrm{N}$, longitude $27^{\circ} 18^{\prime} \mathrm{E}$ ), with an apogee around $27000 \mathrm{~m}$ and the float phase of $4 \mathrm{~h}$. The outdoor temperature during the flight reached $-67{ }^{\circ} \mathrm{C}$; however, the environmental conditions in North Sweden during the complete launch campaign were relatively warm, and no ice cover over the ground was found. During this flight, contributions from four different scattering sources over boreal forests have been observed, coming from the soil, the canopy, and the canopy-soil and soil-trunk interactions. It is found that the coherent reflectivity decreases from $\sim-15$ to $\sim-21 \mathrm{~dB}$ (for the soil), from $\sim-19$ to $\sim-25 \mathrm{~dB}$ (for the canopy), from $\sim-22$ to $\sim-30 \mathrm{~dB}$ (for the canopy-soil), and from $\sim-25$ to $\sim-33 \mathrm{~dB}$ (for the soil-trunk) when elevation angle $\theta_{e}$ increases from $35^{\circ}$ to $72^{\circ}$. The reflected power is nearly independent of the flight height that evidences a strong coherent component for a very high coherent integration time $T_{c}=20 \mathrm{~ms}$. These empirical results show that the scattering over the forest elements occurs in a clear multimodal manner.

\section{ACKNOWLEDGMENT}

The authors would like to thank Mrs. Pons and Mrs. Khoe from the administration of the Department of Signal Theory and Communications (TSC), Universitat Politécnica de Catalunya (BarcelonaTech), Barcelona, Spain, and the Rocket/Balloon EXperiments for University Students (REXUS/BEXUS) teams for their collaboration, help, and advice during the whole project. The authors would also like to thank Dr. N. Callens (ESA) and Mr. A. Kinnaird (Swedish Space Corporation) for their help during the flight campaign, Mr. S. Vey (ESA) for his advice during the thermal analysis, and Dr. F. Fabra [Institute for Space Studies of Catalonia (IEEC)] for his collaboration during the 2013 REXUS/BEXUS Selection Workshop.

\section{REFERENCES}

[1] M. Martín-Neira, "A Passive Reflectometry and Interferometry System (PARIS): Application to ocean altimetry," ESA J., vol. 17, no. 4, pp. 331-355, Jan. 1993.

[2] J. C. Auber, A. Bibaut, and J. M. Rigal, "Characterization of multipath on land and sea at GPS frequencies," in Proc. 7th Int. Tech. Meet. Satell. Div. Inst. Navig., Salt Lake City, UT, USA, Sep. 1994, pp. 1155-1171.

[3] J. L. Garrison and S. J. Katzberg, "Detection of ocean reflected GPS signals: Theory and experiment," in Proc. IEEE Southeastcon, Blacksburg, VA, USA, Apr. 1997, pp. 290-294.

[4] J. L. Garrison, S. J. Katzberg, and M. I. Hill, "Effect of sea roughness on bistatically scattered range coded signals from the global positioning system," Geophys. Res. Lett., vol. 25, no. 13, pp. 2257-2260, Jul. 1998.

[5] F. Fabra, "GNSS-R as a source of opportunity for remote sensing of the cryosphere," Ph.D. dissertation, Dept. Theory Commun., Univ. Politécnica Catalunya, Barcelona, Spain, 2014, (Accessed on 0609 2015). [Online]. Available: www.tdx.cat/bitstream/10803/117605/1/TFFde3.pdf

[6] N. Rodríguez-Alvarez, "Contributions to earth observation using GNSS$R$ opportunity signals," Ph.D. dissertation, Univ. Politécnica Catalunya, Dept. Theory Commun., Barcelona, Spain, 2011, (Accessed on 0410 2015). [Online]. Available: http://www.tdx.cat/handle/10803/53636 
[7] J. L. Garrison, A. Komjathy, V. U. Zavorotny, and S. J. Katzberg, "Wind speed measurement using forward scattered GPS signals," IEEE Trans. Geosci. Remote Sens., vol. 40, no. 1, pp. 50-65, Jan. 2002.

[8] E. Cardellach et al., "Consolidating the precision of interferometric GNSS-R ocean altimetry using airborne experimental data," IEEE Trans. Geosci. Remote Sens., vol. 52, no. 5, pp. 4992-5004, Aug. 2014.

[9] H. Carreno-Luengo, A. Camps, I. Ramos-Pérez, and A. Rius, "Experimental evaluation of GNSS-reflectometry altimetric precision using the $\mathrm{P}(\mathrm{Y})$ and C/A signals," IEEE J. Sel. Topics Appl. Earth Observ. Remote Sens., vol. 7, no. 5, pp. 1493-1500, May 2014.

[10] D. Masters, V. U. Zavorotny, S. Katzberg, and W. Emery, "GPS signal scattering from land for moisture content determination," in Proc. IEEE Int. Geosci. Remote Sens. Symp., Honolulu, HI, USA, Jul. 2000, pp. 3090-3092.

[11] K. M. Larson et al., "Use of GPS receivers as a soil moisture network for water cycle studies," Geophys. Res. Lett., vol. 35, no. 24, no. 24 Dec. 2008, Art. ID L24405.

[12] N. Rodríguez-Alvarez et al., "Soil moisture retrieval using GNSS-R techniques: Experimental results over a bare soil field," IEEE Trans. Geosci. Remote Sens., vol. 47, no. 11, pp. 3616-3624, Nov. 2009.

[13] N. Rodríguez-Alvarez et al., "Soil moisture and vegetation height retrieval using GNSS-R techniques," in Proc. IEEE Int. Geosci. Remote Sens. Symp., Cape Town, South Africa, Jul. 2009, pp. III-869-III-872.

[14] E. E. Small, K. M. Larson, and J. J. Braun, "Sensing vegetation growth with reflected GPS signals," Geophys. Res. Lett., vol. 37, no. 12, Jun. 2010, Art. ID L12401.

[15] A. Egido et al., "Global navigation satellite systems reflectometry as a remote sensing tool for agriculture," Remote Sens., vol. 4, no. 8, pp. 2356-2372, Aug. 2012.

[16] N. Pierdicca et al., "GNSS reflectometry as a tool to retrieve soil moisture and vegetation biomass: Experimental and theoretical activities," in Proc. ICL-GNSS, Torino, Italy, Jun. 2013, pp. 1-5.

[17] N. Pierdicca, L. Guerriero, R. Giusto, M. Brogioni, and A. Egido, "SAVERS: A simulator of GNSS reflections from bare and vegetated soils," IEEE Trans. Geosci. Remote Sens., vol. 52, no. 10, pp. 6542-6554, Oct. 2014.

[18] A. K. Fung and H. J. Eom, "Coherent scattering of a spherical wave from an irregular surface," IEEE Trans. Antennas Propag., vol. AP-31, no. 1, pp. 68-72, Jan. 1983.

[19] N. Pierdicca, L. Guerriero, M. Brogioni, and A. Egido, "On the coherent and non coherent components of bare and vegetated terrain bistatic scattering: Modelling the GNSS-R signal over land," in Proc. IEEE Int. Geosci. Remote Sens. Symp., Munich, Germany, Jul. 2012, pp. 3407-3410.

[20] M. Unwin, P. Jales, P. Blunt, and S. Duncan, "Preparation for the first flight of SSTL's next generation space GNSS receivers," in Proc. 6th ESA/European Workshop Satell. NAVITEC GNSS Signals Signal Process. 2012, pp. 1-6.

[21] S. R. Christopher et al., "The CYGNSS nanosatellite constellation hurricane mission," in Proc. IEEE Int. Geosci. Remote Sens. Symp., Munich, Germany, Jul. 2012, pp. 214-216.

[22] J. Wickert et al., "GEROS-ISS: GNSS reflectometry, radio occultation and scatterometry on-board the international space station," presented at the URSI Commission F Microwave Signatures Specialist Symp. Microwave Remote Sensing Earth, Oceans, Atmosphere, Espoo, Finland, Oct. 2013

[23] M. Martín-Neira, S. D'Addio, C. Buck, N. Floury, and R. Prieto-Cerdeira, "The PARIS ocean altimeter in-orbit demonstrator," IEEE Trans. Geosci. Remote Sens., vol. 49, no. 6, pp. 2209-2237, Jun. 2011.

[24] H. Carreno-Luengo et al., "3Cat-2: A $\mathrm{P}(\mathrm{Y})$ and C/A experimental nanosatellite mission," in Proc. IEEE Int. Geosci. Remote Sens. Symp. Melbourne, Vic., Australia, Jul. 2013, pp. 843-846.

[25] H. Carreno-Luengo, A. Camps, I. Ramos-Pérez, and A. Rius, "A P(Y) \& C/A ReflectOmeter (PYCARO): High resolution sea measurements," presented at the Workshop Reflectometry GNSS Other Signals Opportunity (GNSS+R), West Lafayette, IN, USA, Oct. 2012, (Accessed on 0110 2015). [Online]. Available: http://www.gnssr2012.org/topics/ technical-program

[26] A. Kinnaird, BEXUS User Manual, ID: BX_REF_BX_User Manual v6-10 05, Feb. 14 2014, (Accessed on 0810 2015). [Online]. Available: http://www.rexusbexus.net/index.php?option=com_content\&view $=$ article\&id $=51 \&$ Itemid $=63$

[27] F. T. Ulaby, R. K. Moore, and A. K. Fung, Microwave Remote Sensing: Active and Passive, vol. 2, Radar Remote Sensing and Surface Scattering and Emission Theory. Reading, MA, USA: Addison-Wesley, 1982.

[28] X. Wu and S. Jin, "GPS-reflectometry: forest canopies polarization scattering properties and modelling," Adv. Space Res., vol. 54, no. 5, pp. 863-870, Sep. 2014
[29] J. J. van Zyl, "The effect of topography on radar scattering from vegetated areas," IEEE Trans. Geosci. Remote Sens., vol. 31, no. 1, 153-160, Jan. 1993

[30] P. Ferrazzoli, L. Guerriero, N. Pierdicca, and R. Rahmoune, "Forest biomass monitoring with GNSS-R: Theoretical simulations," Adv. Space Res., vol. 47, no. 10, pp. 1823-1832, May 2011

[31] A. Egido et al., "Airborne GNSS-R soil moisture and above ground biomass observations," IEEE J. Sel. Topics Appl. Earth Observ. Remote Sens., vol. 7, no. 5, pp. 1522-1532, May 2014.

[32] P. Beckmann and A. Spizzichino, The Scattering of Electromagnetic Waves from Rough Surfaces. Norwood, MA, USA: Artech House, 1963.

[33] H. Park et al., "Retracking considerations in spaceborne GNSS-R altimetry," GPS Sol., vol. 16, no. 4, pp. 507-518, Oct. 2012.

[34] A. T. Caicoya, F. Kugler, I. Hajnsek, and K. Papathanassiou, "Boreal forest biomass classification with TANDEM-X," in Proc. IEEE Int. Geosci. Remote Sens. Symp., Munich, Germany, Jul. 2012, pp. 3439-3442.

[35] Global Map of Forest Height Produced from NASA's ICESAT/GLAS, MODIS and TRMM Sensors, (Accessed on 0508 2015). [Online]. Available: http://www.nasa.gov/topics/earth/features/earth20120217map. html

[36] R. D. De Roo and F. T. Ulaby, "Bistatic specular scattering from rough dielectric surfaces," IEEE Trans. Antennas Propag., vol. 42, no. 2, pp. 220-231, Feb. 1994.

[37] H. Park et al., "End-to-end simulator for global navigation satellite system reflectometry space mission," in Proc. IEEE Int. Geosci. Remote Sens. Symp., Honolulu, HI, USA, Jul. 2010, pp. 4294-4297.

[38] T. Elfouhaily, D. R. Thompson, and L. Linstrom, "Delay-Doppler analysis of bistatically reflected signals from the ocean surface: Theory and application," IEEE Trans. Geosci. Remote Sens., vol. 40, no. 3, 560-573, Mar. 2002.

[39] E. Valencia et al., "Experimental determination of the sea correlation time using GNSS-R coherent data," IEEE Geosci. Remote Sens. Lett., vol. 7, no. 4, pp. 675-679, Oct. 2010.

[40] J. Liang and Q. Liang, "Outdoor propagation channel modeling in foliage environment," IEEE Trans. Veh. Technol., vol. 59, no. 5, pp. 2243-2251, Jun. 2010.

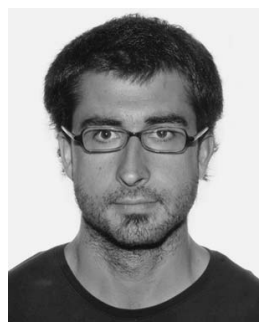

Hugo Carreno-Luengo (S'12-M'14) received the M.St. degree in aeronautical engineering (specialization in spacecraft) from Universidad Politécnica de Madrid, Madrid, Spain, in 2010. He is currently working toward the Ph.D. degree in the Remote Sensing Laboratory, Department of Signal Theory and Communications, Universitat Politécnica de Catalunya (UPC), Barcelona, Spain.

In 2011, he joined the Remote Sensing Laboratory, Department of Signal Theory and Communications, UPC, where is currently provided with a fellowship from the Institute for Space Studies of Catalonia (IEEC). From 2012 to 2015, he was the Team Leader of the Topography From Reflectometric Measurements: An Experiment From the Stratosphere (TORMES) and TORMES 2.0 teams in the frame of the European Space Agency Balloon EXperiments for University Students (BEXUS) 17 and 19 stratospheric flight campaigns. Currently, he is with the UPC team in the European Global Navigation Satellite Systems Reflectometry (GNSS-R) Environmental Monitoring (E-GEM) Seventh Framework Programme (FP7) Project. His research interests include the use of GNSS-R techniques to perform Earth remote sensing from nanosatellites.

Mr. Carreno-Luengo was a Session Chair at the 2015 IEEE International Geoscience and Remote Sensing Symposium. 


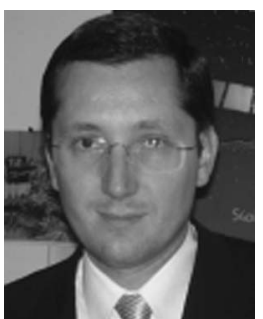

Adriano Camps (S'91-A'97-M'00-SM'03-F'11) was born in Barcelona, Spain, in 1969. He received the Master's and Ph.D. degrees in telecommunications engineering from Universitat Politécnica de Catalunya (UPC), Barcelona, Spain, in 1992 and 1996, respectively.

From 1991 to 1992, he was with École Nationale Supérieure des Télécommunications de Bretagne, Brest, France, with an Erasmus fellowship. Since 1993, he has been with the Remote Sensing Laboratory, Electromagnetics and Photonics Engineering Group, Department of Signal Theory and Communications, UPC, where he was first an Assistant Professor, then an Associate Professor in 1997, and has been a Full Professor since 2007. In 1999, he was on sabbatical leave from the Microwave Remote Sensing Laboratory, University of Massachusetts Amherst, Amherst, MA, USA. Since 1993, he has been deeply involved in the European Space Agency Soil Moisture and Ocean Salinity (SMOS) Earth Explorer Mission, from the instrument and algorithmic points of view, performing field experiments and more recently studying the use of global navigation satellite systems reflectometry (GNSS-R) techniques to perform the sea-state correction needed to retrieve salinity from radiometric observations. He is the author or coauthor of nearly 100 papers in peer-reviewed journals and more than 200 international conference presentations. His research interests are focused on microwave remote sensing, with special emphasis on microwave radiometry by aperture synthesis techniques and remote sensing using signals of opportunity (GNSS-R).

Dr. Camps was the Chair of u Cal in 2001, a Technical Program Committee Cochair of the 2007 IEEE International Geoscience and Remote Sensing Symposium, and a Cochair of the 2010 GNSS-R. Currently, he is an Associate Editor of Radio Science and the IEEE TRAnsactions on Geoscience And Remote Sensing, and he is the President and Founder of the IEEE Geoscience and Remote Sensing Society Chapter in Spain. He was the recipient of the Second National Award of University Studies in 1993, the INDRA Award of the Spanish Association of Telecommunication Engineers for the best $\mathrm{Ph} . \mathrm{D}$. in remote sensing in 1997, the Extraordinary Ph.D. Award from UPC in 1999, the Research Distinction of Generalitat de Catalunya for contributions to microwave passive remote sensing in 2002, the European Young Investigator Award in 2004, and the Catalan Institution for Research and Advanced Studies (ICREA) Academia Award in 2009. Moreover, as a member of the Microwave Radiometry Group, UPC, he was the recipient of the First Duran Farell Award in 2000, the Ciutat de Barcelona Award for Technology Transfer in 2001, the "Salvá i Campillo" Award of the Professional Association of Telecommunication Engineers of Catalonia for the most innovative research project for Microwave Imaging Radiometer with Aperture Synthesis/SMOS-related activities in 2004, and the Seventh Duran Farell Award for Technological Research for the work on GNSS-R instrumentation and applications in 2010.

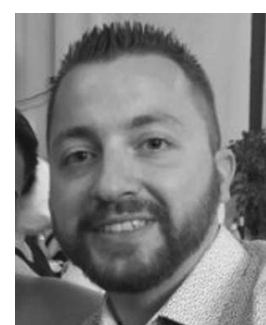

Jorge Querol (S'14) was born in Forcall, Spain, in 1987. He received the B.S. degree in electronics engineering and the B.S. degree in telecommunication engineering from Universitat Politécnica de Catalunya (UPC), Barcelona, Spain, in 2011 and 2012, respectively. He is currently working toward the M.S. degree in photonics in the Remote Sensing Laboratory, Department of Signal Theory and Communications, UPC, working on his M.S. thesis implementing real-time wavelet-based radio frequency interference mitigation algorithms for microwave radiometry applications.

In summer of 2010, he did an internship with the Institut de Ciéncies Fotóniques, Castelldefels, Spain, where he developed signal processing algorithms for medical imaging. From 2011 to 2012, he was with Kungliga Tekniska Högskolan, Stockholm, Sweden, with an Erasmus fellowship. During this time, he collaborated with the Network Systems Laboratory, working with control algorithms for efficient energy storage and distribution systems.

Mr. Querol was the recipient of the Lear Corporation Award for the best academic records of the year in electronics engineering in 2012.

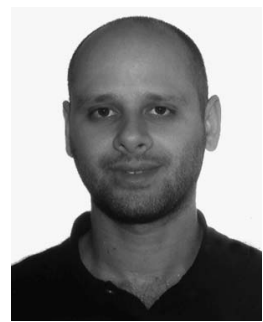

Giuseppe Forte (M'11) was born in Guatemala City, Guatemala, in 1979. He received the M.St. degree in electronics engineering from Universidad Francisco Marroquín, Guatemala City, Guatemala, in 2002 and the Advanced Studies in Electronic Engineering Diploma from Universitat Rovira i Virgili, Tarragona, Spain, in 2005. He is currently working toward the Ph.D. degree in telecommunications engineering at Universitat Politécnica de Catalunya (UPC), Barcelona, Spain.

Since 2010, he has been involved with the Remote Sensing Laboratory, Department of Signal Theory and Communications, UPC, performing the design and implementation of algorithms, electronics, and field experiments related to remote sensing and radio frequency interference detection and mitigation. 